\title{
Onchocerciasis in Ecuador: evolution of chorioretinopathy after amocarzine treatment
}

\author{
P J Cooper, R Proaño, C Beltran, M Anselmi, R H Guderian
}

\begin{abstract}
Aims-To investigate the impact of the macrofilaricidal drug, amocarzine, on the evolution of chorioretinopathy in onchocerciasis.

Methods-A prospective uncontrolled cohort study was performed using subjects infected with Onchocerca volvulus in a hyperendemic onchocerciasis focus in Esmeraldas Province in Ecuador. Study subjects were recruited into four cohorts in which ophthalmic and parasitological data were collected for $2,3,4$, and 5 years respectively.

Results-Complete ophthalmic follow up was obtained for 294 individuals in the four cohorts. The incidence of retinal pigment epithelial atrophy tended to remain constant between cohorts while that of chorioretinal scarring with a greater observation period. The incidence rate of cases with new or extending chorioretinal lesions was greater with an increasing period of follow up. An association was seen between the cumulative microfilarial loads in the skin and the development of new chorioretinal lesions $(p<0 \cdot 05)$. No relation was noted between cumulative microfilarial loads and the progression of existing disease.

Conclusion-Amocarzine therapy did not prevent the natural evolution of chorioretinal disease. It was suggested that ocular microfilariae were necessary for the induction of chorioretinopathy in previously unaffected eyes and that extension of existing disease might also be related to the presence of ocular microfilariae or to other immunological mechanisms.

(Br F Ophthalmol 1996; 80: 337-342)
\end{abstract}

Onchocerciasis is a major blinding disease in parts of Africa, the Yemen, and Central and South America. The parasite, Onchocerca volvulus, is a filarial nematode which is transmitted by blackflies of the genus Simulium. It infects an estimated 18 million people worldwide of which about 350000 are blind and a similar number are visually impaired. ${ }^{1}$

Blinding lesions may affect the anterior and posterior segments and include sclerosing keratitis, iridocyclitis, optic atrophy, and chorioretinopathy. ${ }^{2}$ Posterior segment lesions, particularly chorioretinopathy, account for most visual loss in this disease. ${ }^{3-5}$

Amocarzine is an isothiocyanate derivative with both macrofilaricidal ${ }^{6}$ and microfilaricidal effects. ${ }^{7}$ The clinical efficacy of this compound in onchocerciasis has been demonstrated in field trials in Ecuador ${ }^{8}$ and Guatemala. ${ }^{9}$ There are no published reports of the long term effects of this promising macrofilaricide on posterior segment disease. This is of particular importance in the light of the well documented adverse effects of other filaricidal drugs on the posterior segment. ${ }^{1011}$

The natural history of onchocercal chorioretinopathy is poorly understood. Most epidemiological studies which have examined this process have linked its presence with skin and ocular microfilarial indices. ${ }^{12-14}$ However, onchocercal chorioretinopathy is a chronic progressive inflammatory process ${ }^{3}$ that continues to progress despite the reduction of microfilarial levels by chemotherapy ${ }^{31516}$ or vector control. ${ }^{17}$ This latter observation has generated renewed interest into the pathogenesis of the lesion. ${ }^{18-20}$

This study examined longitudinally the relation between the evolution of chorioretinopathy and detailed changes in microfilarial burdens in $O$ volvulus infected subjects who had received the oral macrofilaricide amocarzine.

\section{Materials and methods}

STUDY POPUEATION

Patients were recruited from 33 villages in the hyperendemic area in the rain forest focus on onchocerciasis in the Santiago river basin of Esmeraldas Province in Ecuador. The communities living in this area included both indigenous Amerindian (Chachi tribe) and Ecuadorians of African descent (AfroEcuadorians). The ethnographic features of these communities have been described elsewhere. $^{21}$

Patients were selected in four separate cohorts starting in February 1988, March 1989, March 1990, and March 1991 and were followed up for $5,4,3$, and 2 years respectively. All study participants were volunteers who were known to have palpable nodules and microfilariae in skin snips from a previous survey. The different cohorts received different regimens of oral amocarzine as part of a study to investigate the efficacy, tolerability, and pharmacokinetics of amocarzine and to optimise dosage regimens. ${ }^{8}$ Amocarzine was administered biannually as follows: 1988, 1990 , and 1992 in the 5 year cohort; 1989 and 1991 in the 4 year cohort; 1990 and 1992 in the 3 year cohort; and 1991 in the 2 year cohort. The total dose administered to each cohort is shown in Table 1 . Selection of the first cohort was restricted to males with moderate skin microfilarial (mf) counts (range 30-50 $\mathrm{mf} / \mathrm{mg}$ ), while microfilarial intensity did not 
Table 1 The incidence of retinal pigment epithelial atrophy and chorioretinal scarring in the four cohorts

\begin{tabular}{llclccccc}
\hline Cohort & $\begin{array}{l}\text { Dose } \\
(\mathrm{mg} / \mathrm{kg})\end{array}$ & $n$ & $\begin{array}{l}R P E \\
\text { first }\end{array}$ & $\begin{array}{l}\text { RPE } \\
\text { last }\end{array}$ & $\begin{array}{l}\text { Incident } \\
R P E(\%)\end{array}$ & $\begin{array}{l}\text { Scar } \\
\text { first }\end{array}$ & $\begin{array}{l}\text { Scar } \\
\text { last }\end{array}$ & $\begin{array}{l}\text { Incident } \\
\text { scar (\%) }\end{array}$ \\
\hline 5 Years & 38 & 16 & 2 & 3 & $1(6)$ & 3 & 8 & $5(31)$ \\
4 Years & 36 & 102 & 5 & 19 & $14(14)$ & 16 & 42 & $26(25)$ \\
3 Years & 38 & 52 & 1 & 3 & $2(4)$ & 7 & 13 & $4(12)$ \\
2 Years & 18 & 124 & 8 & 14 & $6(5)$ & 11 & 18 & $3(6)$ \\
\hline
\end{tabular}

Dose $=$ total dose of amocarzine administered during the observation period: $n=$ number of subjects in each cohort; RPE first=number of subjects with evidence of atrophy of the retinal pigment epithelium at the first examination; RPE last=number of subjects with evidence of pigment epithelium at the first examination; RPE last $=$ number of subjects with evidence of
atrophy of the retinal pigment epithelium at the last examination; Scar first $=$ number of subjects with evidence of chorioretinal scarring at the first examination; Scar last=number of subjects with evidence of chorioretinal scarring at the last examination.

influence selection of the remaining three cohorts. Selection of each cohort was performed without reference to ocular status.

Parasitological evaluation of skin and ocular microfilarial intensities was performed annually with the last examination in March 1993 for all groups. Detailed examinations of retinal disease were performed at the first and last examination times (for example, at the start of each cohort and in March 1993).

\section{PARASITOLOGICAL EXAMINATION}

Skin biopsies were taken from both iliac crests with a Stolz corneoscleral punch. The biopsies were weighed and placed in micro well plates in physiological saline. The numbers of emergent microfilariae were counted after 24 hours. The geometric mean of the two snips was calculated for each person.

\section{OPHTHALMIC EXAMINATION}

Slit-lamp examination of the anterior segment and measurement of intraocular pressures were performed as previously described. ${ }^{22}$ After pupil dilatation with $1 \%$ tropicamide and $10 \%$ phenylephrine, the ocular fundus was examined with a Keeler indirect ophthalmoscope. The optic disc was examined for the presence of atrophy, papillitis, increased pigmentation, and sheathing of the central retinal vessels. Abnormalities of the retina and choroid were noted. Particular attention was paid to the presence of mottling or confluent atrophy of the retinal pigment epithelium (RPE) and chorioretinal scarring with pigment clumping and/or choriocapillary atrophy.

\section{ASSESSMENT OF CHORIORETINAL CHANGES}

Fundal observations were recorded pictorially for each eye by dividing the eye into quadrants with two diagonal lines which bisected at the macula. The development of new chorioretinal lesions or extension of old lesions were recorded per person. Chorioretinal changes were classified into two types: (1) new lesions (type 1) included those with no evidence of chorioretinopathy in either eye at the first examination and who developed chorioretinal lesions during the period of observation; and (2) progression of existing disease (type 2) included all those with chorioretinopathy at the first examination in whom the disease progressed over the period of observation and included symmetrical new lesions (chorioretinopathy in the contralateral eye at the first examination but developed new lesions in a previously normal eye), extension of existing disease (chorioretinopathy in one or both eyes at the first examination and developed either new lesions in the affected eye or the existing lesions extended geographically beyond the affected quadrant), or a combination of these.

Ocular examinations were performed by two experienced ophthalmologists (RP and $\mathrm{CB}$ ). No systematic test of between and within observer variation was performed.

\section{STATISTICAL ANALYSIS}

Infection intensities of microfilariae in the skin and anterior segment are expressed as the geometric mean number of microfilariae per individual. Skin infection intensities are expressed as microfilariae per $\mathrm{mg}$ of skin. Comparison of proportions was analysed using $\chi^{2}$ and of means using Student's $t$ test for two independent groups and one way analysis of variance for more than two groups. Cumulative incidence rates were calculated for each cohort as the ratio of the number of new cases with chorioretinopathy to the total population at risk. Spearman's rank correlation coefficient was used to calculate the correlation between two variables. The relation between chorioretinopathy and microfilarial levels was further analysed by multiple logistic regression (GLIM, Numerical Algorithms Group, Oxford). The effect of a single variable was assessed by comparing scaled deviances between the model with the variable fitted and the model without the fitted variable. The differences in deviance had a $\chi^{2}$ distribution with the numbers of degrees of freedom that differed between the two models. ${ }^{23}$ As microfilarial counts in the skin and anterior chamber declined in all cohorts over the observation periods, an index of cumulative microfilarial burden was calculated for each individual as the area under the curve (AUC) of the relation between microfilarial infection intensity and observation period. AUCs were calculated using the trapezium rule ${ }^{24}$ and logarithmically transformed to normalise the data for parametric statistical analysis.

\section{Results}

\section{STUDY POPULATION}

A total of 369 individuals were recruited into the four cohorts. Of these $294(79 \cdot 7 \%)$ were followed completely and are included in the analysis. The age distribution of the four cohorts is shown in Table 2.

A total of $75(20.3 \%)$ individuals of the 369 starting the study were lost to follow up. These individuals left the study because of emigration $(62 / 75)$ from the study area or death (10/75). Loss of data excluded the remaining three from the analysis. Loss to follow up affected $33.3 \%$ of the 5 year cohort, $15 \cdot 7 \%$ of the 4 year cohort, $30.6 \%$ of the 3 year cohort, and $16.8 \%$ of those followed for 2 years. There 
Table 2 Age distribution of the four cohorts

\begin{tabular}{lcccr}
\hline \multirow{4}{*}{$\begin{array}{l}\text { Age } \\
\text { group }\end{array}$} & \multicolumn{4}{l}{ Cohort (\%) } \\
\cline { 2 - 5 } & 5 Years & 4 Years & 3 Years & 2 Years \\
\hline $0-19$ & $0(0)$ & $1(1 \cdot 0)$ & $16(30 \cdot 8)$ & $41(33 \cdot 1)$ \\
$20-29$ & $5(31 \cdot 3)$ & $20(19 \cdot 6)$ & $21(40 \cdot 4)$ & $36(29 \cdot 0)$ \\
$30-39$ & $7(43 \cdot 8)$ & $23(22 \cdot 6)$ & $4(7 \cdot 7)$ & $23(18 \cdot 5)$ \\
$40-49$ & $1(6 \cdot 3)$ & $24(23 \cdot 5)$ & $10(19 \cdot 2)$ & $16(12 \cdot 9)$ \\
$50+$ & $3(18 \cdot 6)$ & $34(33 \cdot 3)$ & $1(1 \cdot 9)$ & $8(6 \cdot 5)$ \\
Total & $16(100)$ & $102(100)$ & $52(100)$ & $124(100)$ \\
\hline
\end{tabular}

were no systematic differences between those individuals lost to follow up and those included in the analysis in terms of age, race, sex, prevalence of chorioretinopathy, and microfilarial intensities in the skin and anterior chamber.

\section{MICROFILARIAL INTENSITIES IN THE SKIN AND}

EYE

MfS and MfAC levels were documented annually in all cohorts and changes with time are shown in Figures 1 and 2. There was marked variation in both microfilarial indices at the first examination between the four cohorts (MfS p<0.001; MfAC $\mathrm{p}<0.001$ ). MfS levels declined immediately following treatment in all groups except for the 4 year cohort where a decline in intensity was only detected immediately before the second dose of amocarzine (after a 2 year delay) (Fig 1). MfS counts had fallen to below $2 \mathrm{mf} / \mathrm{mg}$ in all groups by March 1993. MfAC levels also declined following treatment but less dramatically (Fig 2). By the final examination, MfAC levels had fallen to a mean of $0.1 \mathrm{mf} /$ person per eye for all groups. There were no significant differences between the cohorts in levels of MfS and MfAC at the final observation.

Cumulative microfilarial loads are an index of the number of skin and anterior chamber microfilariae harboured by each individual over their respective periods of observation. Geometric mean cumulative skin microfilarial burdens were $124 \cdot 6,39 \cdot 3,28 \cdot 3$, and $29 \cdot 3$ for the $5,4,3$, and 2 year cohorts, respectively. The equivalent cumulative microfilarial burdens in the anterior chamber were $7 \cdot 5,2 \cdot 3$, $1 \cdot 2$, and $2 \cdot 1$, respectively. Cumulative skin $(p<0.001)$ and anterior chamber $(p<0.001)$ burdens of microfilariae were significantly different between cohorts.

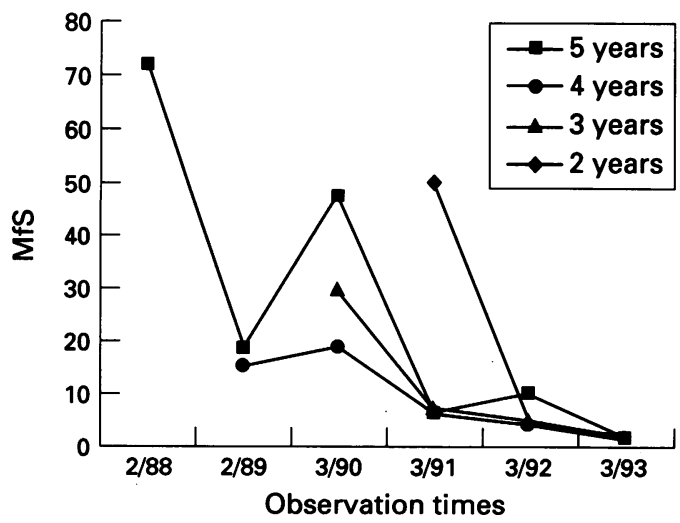

Figure 1 Skin microfilarial intensities (MSS) in the four cohorts over the follow up periods. Times of amocarzine administration are given in the text.

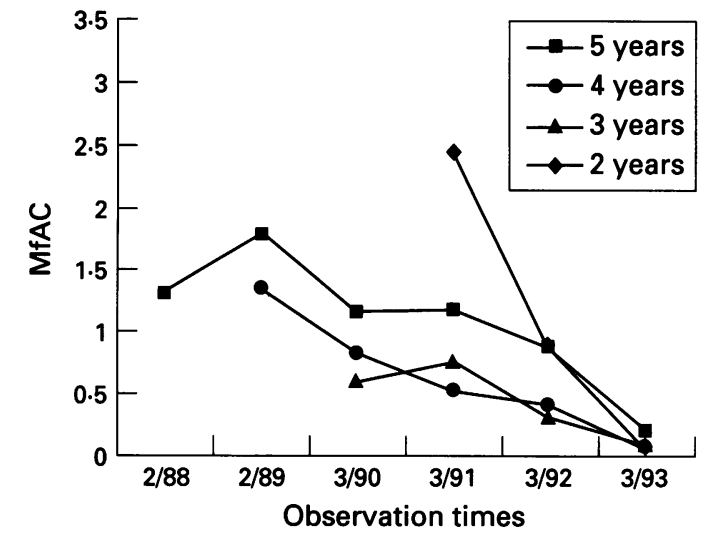

Figure 2 Anterior chamber microfilarial intensities (MfAC) in the four cohorts over the follow up periods. Times of amocarzine administration are given in the text.

INCIDENCE OF CHORIORETINOPATHY

The incidence of RPE atrophy and chorioretinal scarring in the four cohorts is shown in Table 1. The incidence of RPE atrophy remained remarkably constant in all four cohorts. However, the 4 year cohort had a much higher incidence of RPE atrophy than the other three cohorts. The incidence of chorioretinal scarring increased with greater period of follow up.

\section{DEVELOPMENT OF NEW AND EXTENSION OF} OLD CHORIORETINAL LESIONS

These were seen in $97(33.0 \%)$ individuals; new lesions (type 1) developed in 68 individuals and progression of existing disease (type 2) was seen in 29 individuals ( 16 developed symmetrical new lesions, six showed evidence of extension of an existing lesion, and seven showed a combination of these).

The incidence rates for both types of lesions are shown in Table 3. The risk of developing new lesions (type 1) was greater with increasing length of follow up. The risk of progression of existing chorioretinal disease (type 2) also increased with length of follow up but was greater in the 4 year than the 5 year cohort. The risk of disease progression (type 2) was greater than that of developing a new lesion (type 1) in all cohorts.

Resolution of chorioretinal lesions was seen in one individual in the 2 year cohort $(0.8 \%)$. This took the form of the disappearance of early temporal RPE atrophy.

RELATION BETWEEN MICROFILARIAL INFECTION INTENSITIES AND CHORIORETINOPATHY

New lesions (type 1)

Cumulative intensities in the skin $(p<0.05)$ and eye $(p<0.05)$ correlated with the development of new chorioretinal lesions. The development of new lesions was strongly associated with both age $(p<0.001)$ and period of follow up $(p<0.001)$. No association with race or sex was seen. After controlling for age and cohort, an association remained between cumulative skin infection intensity and the development of new chorioretinal lesions $(p<0.01)$. 
Table 3 Cumulative incidence rates in each cohort for the number of cases either developing new chorioretinal lesions (type 1) or showing progression of existing lesions (type 2)

\begin{tabular}{lcclcccl}
\hline Cohort & $\begin{array}{l}\text { Number } \\
\text { at risk }\end{array}$ & $\begin{array}{l}\text { Type } \\
1\end{array}$ & $\begin{array}{l}\text { Incidence } \\
\text { (\%) }\end{array}$ & $\begin{array}{l}\text { Number } \\
\text { at risk }\end{array}$ & $\begin{array}{l}\text { Type } \\
2\end{array}$ & $\begin{array}{l}\text { Incidence } \\
(\%)\end{array}$ \\
\hline 5 Years & 11 & 6 & 55 & 5 & 4 & 80 \\
4 Years & 81 & 40 & 49 & 21 & 19 & 90 \\
3 Years & 44 & 8 & 18 & 8 & 3 & 38 \\
2 Years & 105 & 14 & 13 & 19 & 3 & 16 \\
\hline
\end{tabular}

Number at risk=number of individuals at risk of developing the specified lesion in each cohort.

\section{Progression of existing lesions (type 2)}

There were no significant correlations between any of the indices of microfilarial intensity and individuals showing extension of existing chorioretinal lesions. Progression of existing chorioretinal disease was strongly associated with longer period of follow up $(p<0.001)$, but not age, race, or sex.

\section{Discussion}

Amocarzine therapy was associated with progression of onchocercal chorioretinopathy. However, it is difficult to dissociate possible adverse drug effects from the natural evolution of chorioretinopathy as no control or placebo group was included. Treatment with other filaricidal drugs such as ivermectin, ${ }^{325-27}$ (Whitworth J, personal communication; Murdoch I, personal communication) diethylcarbamazine, ${ }^{1011}$ and suramin ${ }^{1016}$ does not prevent evolution of chorioretinopathy. Evolution of chorioretinopathy was seen with a similar frequency in those treated with ivermectin as in those receiving placebo. ${ }^{3}$ (Whitworth $\mathrm{J}$, personal communication; Murdoch I, personal communication) In this study, it is possible that some new lesions resulted from a direct effect of amocarzine. Retinal changes directly attributable to treatment with microfilaricides (diethylcarbamazine and ivermectin) are generally seen within a few days of drug administration and take the form of transient window defects in the RPE on fluorescein angiography. 112829 Angiographic studies performed up to 1 year after administration of amocarzine remained normal, ${ }^{9}$ though early transient changes might have been missed. In this study, all study participants were re-examined 1 week after amocarzine administration at each treatment time and none of the lesions documented was first noted at this time point.

The method by which disease was recorded was subject to error which was minimised by using two experienced ophthalmologists (RP and $\mathrm{CB}$ ), each of whom examined the same patients over the study period. Only one individual was recorded as having an improvement in chorioretinal disease which suggests that the fundal findings were accurately and consistently reported. This and other studies 312 have demonstrated that onchocercal chorioretinopathy is progressive and evidence of disease resolution is generally only seen in the transient post-treatment defects in the RPE described above.

The chorioretinal lesions seen in this onchocerciasis focus ${ }^{22}$ are identical to those described elsewhere as typical of onchocerciasis. $^{3} 30$ The discrete pigmented atrophic scars of ocular toxoplasmosis were recorded in 11 (or $4 \%$ ) of the study participants (data not shown) of which seven had evidence of concurrent onchocercal chorioretinopathy. The latter was characterised by diffuse, pigmented chorioretinal scars associated with RPE atrophy. Mottling of the RPE attributable to ocular toxoplasmosis is a rare findings. ${ }^{31} 32$ No abnormalities of the retina attributable to other disease processes were recorded.

High incidence rates of new chorioretinal lesions and extension of existing disease were seen in this study. Two other longitudinal studies have attempted to estimate the cumulative incidence for the development and deterioration of posterior segment lesions: Anderson and co-workers ${ }^{13}$ followed up communities in Cameroon over 4 years and estimated the incidence of development/deterioration of chorioretinopathy to be $6.7 \%$ in the rain forest and $4.2 \%$ in the savanna. Between villages this risk varied from 0 to $23 \%$ in both regions. ${ }^{33}$ The second study followed 263 individuals (suramin or no treatment) for 14-15 years in the Nigerian savanna. ${ }^{12}$ From data available in this report, incidence risks for the development/deterioration of posterior segment lesions can be calculated; extension of existing lesions (type 2) was seen in $45 \cdot 2 \%$ of untreated and $52 \cdot 6 \%$ of treated subjects and new lesions (type 1 ) in $24.8 \%$ of the untreated and $13.8 \%$ of those treated. These estimates are considerably less than the equivalent estimates obtained in this study (comparing the 4 year cohort with the first study and the 5 year cohort with the second study).

The high incidence of chorioretinal lesions seen in this study (Table 1) might be explained by the changes in epidemiology that have occurred since 1980 in this focus. ${ }^{34-36}$ Large increases in the prevalence and intensity of infection were seen between 1980 and $1986 .^{34}$ This is thought to have followed large increases in transmission that followed the $\mathrm{El} \mathrm{Nino}$ climatic disturbances of 1982 to $1984 .{ }^{35}$ The high incidence of chorioretinopathy may be a consequence of this shift in endemicity which occurred over a relatively short period of time. This might, for example, have resulted in enhanced clinical pathology as seen in immunologically naive immigrants/volunteers. ${ }^{37}$ Alternatively, the high incidence of chorioretinal disease seen during the study period (1987-93) might be a result of the natural time lag expected between ocular parasitisation, which would be more prevalent and intense, and the induction of retinal pathology.

A number of longitudinal studies have suggested an association between the presence of either MfS and/or MfAC and the development of new lesions, ${ }^{12} 14$ but none of these attempted to quantify the intensity of infection or the magnitude of the association. The single study that did found a strong relation between microfilarial intensities in the skin and anterior chamber and the deterioration or development of chorioretinal lesions in the rain forest of Cameroon. ${ }^{13}$ The use of an index of cumulative microfilarial load intuitively seems a better 
index of infection intensity than single measurements which are often highly variable. This was particularly important in this study because of the long term reduction in microfilarial burdens that followed amocarzine therapy.

Microfilarial counts in the skin seem to be more important than those in the anterior chamber. ${ }^{13}$ This may simply reflect the larger error involved in the assessment of anterior chamber microfilariae as the accuracy of assessment is strongly dependent on the cooperation of the patient both before and during examination (and the skill of the observer). A number of workers have demonstrated that the level of ocular parasitisation is strongly associated with the development of new chorioretinal lesions. ${ }^{12} 13$ The presence of head nodules is also a useful index of risk ${ }^{38}$ and following nodulectomy, only those with greater than 15 microfilariae at the outer canthus developed new chorioretinal lesions. ${ }^{39}$ The presence of microfilariae in vitreous has also been associated with development of chorioretinopathy. ${ }^{13}$ There can be little doubt, therefore, that ocular microfilariae are essential for the induction of chorioretinal lesions. Retinal damage would result from the inflammatory reactions that follow the death of intraretinal microfilariae. The finding of RPE disturbances in a classically onchocercal distribution (for example, the temporal edge of the macula) soon after administration of diethylcarbamazine ${ }^{11}$ strongly supports this hypothesis.

Less clear, however, is the pathogenesis of extending chorioretinal disease. The mechanism could be as described for new lesions. Extension of existing lesions has been described in untreated populations ${ }^{12-14}$ and following chemotherapy 15162527 and vector control. ${ }^{17} 40$ Extension of existing chorioretinopathy can occur within a few days of administration of diethylcarbamazine, ${ }^{11}$ but generally progresses slowly over years. ${ }^{3}$ In this study, there was no relation between extension of existing chorioretinal lesions and indices of infection intensity. This may also be true of slowly progressive disease following effective chemotherapy and vector control, where ocular and dermal microfilarial intensities are significantly reduced. In addition, progression occurs at the same rate in ivermectin and mebendazole treated patients as in those receiving placebo. ${ }^{3}$ This evidence suggests that the microfilarial density does not influence progression of existing disease. It is possible that the induction of pathology might set off self-perpetuating autoimmune processes that no longer require the presence of microfilariae. Such pathology would only affect those individuals who had already developed microfilariae induced chorioretinal lesions. The evidence for a role for autoimmunity in onchocercal chorioretinopathy is conflicting: an association between the presence of retinal antibodies and chorioretinal disease has been demonstrated by some $\mathrm{e}^{2041}$ but not others ${ }^{18}$ and there is no evidence of specific $T$ cell autoreactivity. 1842
We thank Tony Fulford for statistical advice and Tom Nutman for helpful comments on the manuscript. The work was supported by Christoffel Blindenmission and the Onchocerciasis Project, Hospital Vozandes. PC was supported by a training award from the British Medical Research Council and a Sackler studentship. Keeler Ltd is thanked for the loan of an indirect ophthalmoscope and the British Council for the Prevention of ophthalmoscope and the British Cound
Blindness for a grant to purchase it.

1 World Health Organisation Expert Committee on Onchocerciasis, third report. Technical Report Series 752. Geneva: WHO, 1987.

2 Thylefors B. Ocular onchocerciasis. Bull WHO 1978; 56: 63-78.

3 Semba RD, Murphy RP, Newland HS, Awadzi K, Greene BM, Taylor HR. Longitudinal study of lesions of the posterior segment in onchocerciasis. Ophthalmology 1990; 97: terior seg

4 Newland HS, White AT, Greene BM, Murphy RP, Taylor HR. Ocular manifestations of onchocerciasis in a rain forest area of West Africa. Br $\mathcal{F}$ Ophthalmol 1991; 75: 163-9.

5 Abiose A, Murdoch I, Babalola O, Cousens S, Liman I, Onyema J, et al. Distribution and aetiology of blindness and visual impairment in mesoendemic onchocercal communities, Kaduna State, Nigeria. Br f Ophthalmol 1994; 78: 8-13.

6 Striebel HP, Gallay J, Gelzer J, Jenny EF, Rao VR, Reddy $\mathrm{AB}$, et al. Three new chemotherapeutic agents with potent AB, et al. Three new chemotherapeutic agents with pote

7 Soula G, Pichard E, Doumbo O, Soula E, Poltera A, Striebel HP. Activity of thiocarbamazine in patients dually infected with Schistosoma and Onchocerca volvulus. Lancet 1989; 1: 726 .

8 Guderian RH, Anselmi M, Proaño R, Naranjo A, Poltera AA, Moran M, et al. Onchocercicidal effect of three drug regimens of amocarzine in 148 patients of two races and both sexes from Esmeraldas, Ecuador. Trop Med Parasitol 1991; 52: 263-85.

9 Zea-Flores G, Beltranena F, Poltera AA, Lopez M, Moran $\mathrm{M}$, Zea-Flores CE, et al. Amocarzine investigated as oral onchocercicidal drug in 272 adult male patients from onchocercicial drug in 272 adult male patients from Guatemala. Results from three dose regimens spr
three days. Trop Med Parasitol 1991; 42: 240-62.

10 Anderson J, Fuglsang H. Further studies on the treatment of ocular onchocerciasis with diethylcarbamazine and suramin. Br $\mathcal{F}$ Ophthalmol 1978; 62: 450-7.

11 Bird AC, El Sheikh H, Anderson J, Fuglsang H. Changes in visual function and in the posterior segment of the eye during treatment of onchocerciasis with diethylcarbamazine citrate. Br $\mathcal{F}$ Ophthalmol 1980; 64: 191-200.

12 Budden FH. The natural history of ocular onchocerciasis over a period of 14-15 years and the effect on this of a single course of suramin therapy. Trans $R$ Soc Trop Med Hyg 1976; 70: 484-91.

13 Anderson J, Fuglsang H, Marshall TF de C. Studies on onchocerciasis in the United Cameroon Republic. A four year follow-up of 6 rain-forest and 6 savanna villages. Trans $R$ Soc Trop Med Hyg 1976; 70: 362-73.

14 Rolland A, Thylefors B, Pairault C. Evolution sur 9 ans de l'onchocercose oculaire dans une communaute villageoise d'Afrique occidentale. Bull Organ Mond Sante 1978; 56: 805-10.

15 Anderson J, Fuglsang H, Marshall TF de C. Effects of diethylcarbamazine on ocular onchocerciasis. Tropenmed Parasitol 1976; 27: 263-78.

16 Anderson J, Fuglsang H, Marshall TF de C. Effects of suramin on ocular onchocerciasis. Tropenmed Parasitol 1976; 27: 279-96.

17 Dadzie KY, Remme J, Rolland A, Thylefors B. The effect of 7-8 years of vector control on the evolution of ocular onchocerciasis in West African savanna. Trop Med Parasitol 1986; 37: 263-70.

18 Van der Lelij A, Rothova A, Stilma JS, Vetter JCM, Hoekzema R, Kijlstra A. Humoral and cell-mediated immune response against human retinal antigens in relation to ocular onchocerciasis. Acta Leidensia 1990; 51: 271-83.

19 Braun G, McKechnie NM, Connor V, Gilbert CE, Engelbrecht F, Whitworth JA, et al. Immunological crossreactivity between a cloned antigen of Onchocerca volvulus and a component of the retinal pigment epithelium f Exp Med 1991; 174: 169-77.

20 McKechnie NM, Braun G, Connor V, Klager S, Taylor $\mathrm{DW}$, Alexander RA, et al. Immunologic cross-reactivity in the pathogenesis of ocular onchocerciasis. Invest the pathogenesis of ocular oncho

21 Guderian RH, Leon LA, Leon R, Corral F, Vasconez C, Juderian RH, Leon LA, Leon R, Corral F, Vasconez C,
Johnston TS. Report on a focus of onchocerciasis of Esmeraldas province of Ecuador. Am $\mathcal{F}$ Trop Med Hyg 1982; 31: 270-4.

22 Cooper PJ, Proaño R, Beltran C, Anselmi M, Guderian RH. Onchocerciasis in Ecuador: ocular findings in Onchocerca volvulus infected individuals. $\mathrm{Br} F$ Ophthalmol 1995; 79: 157-62.

23 McCullagh P, Nelder JA. Generalised linear models. Monographs on statistics and applied probability No 37. London: Chapman and Hall, 1992: 118-9.

24 Altman DG. Practical statistics for medical research. London: Chapman and Hall, 1991: 431-3.

25 Rothova A, Van der Lelij A, Stilma JS, Klaassen-Broekema $\mathrm{N}$, Wilson WR, Barbe RF. Ocular involvement in patients with onchocerciasis after repeated treatment with ivermectin. Am $f$ Ophthalmol 1990; 110: 6-16. 
26 Dadzie KY, Remme J, Alley ES, de Sole G. Changes in ocuar onchocerciasis four and twelve months after community-based treatment with ivermectin in a holoendemic onchocerciasis focus. Trans $R$ Soc Trop Med Hyg 1990; 84: onchocer.

27 World Health Organisation. The effect of repeated ivermectin treatment on ocular onchocerciasis; report of an informal consultation. TDR/TDE/ONCHO/93.3, 1993.

28 World Health Organisation. The pathogenesis and treatment of ocular onchocerciasis. TDR/FIL $/ S W G(8) / 82.3$, 1982

29 Taylor HR, Murphy RP, Newland HS, White AT, D'Anna SA, Keyvan-Larijani E, et al. Treatment of onchocerciasis. The ocular effects of ivermectin and diethylcarbamazine. Arch Ophthalmol 1986; 104: 863-70.

30 Bird AC, Anderson J, Fuglsang H. Morphology of posterior segment lesions of the eye in patients with onchociasis. segment lesions of the eye in patien

31 Friedman CT, Knox DL. Variations in recurrent active toxoplasmosis retinochoroiditis. Arch Ophthalmol 1969; 81: 481-93.

32 Doft BH, Gass JD. Outer retinal layer toxoplasmosis. Graefes Arch Clin Exp Ophthalmol 1986; 224: 78-82.

33 Anderson J, Fuglsang H, Marshall TF de C, Radolowicz A, Vaughan JP. Studies on onchocerciasis in the United Cameroon Republic. IV. A four-year follow-up of six rainforest and six savanna villages. The incidence of ocular lesions. Trans $R$ Soc Trop Med Hyg 1978; 72: 513-5.

34 Guderian RH, Beck BJ, Proano SR, Mackenzie CD Onchocerciasis in Ecuador, 1980-1986: epidemiological evaluation of the disease in the Esmeraldas Province. Eur I Epidemiol 1989; 5: 294-302.

35 Guderian RH, Beck BJ, Proano SR. Onchocerciasis in Ecuador: infection in children in the Santiago Basin focus, Province of Esmeraldas. Trans R Soc Trop Med Hyg 1990; 84: 109-12.

36 Guderian RH, Shelley AJ. Onchocerciasis in Ecuador: the situation in 1989. Mem Inst Oswaldo Cruz 1992; 87: 405-15.

37 Rodger FC. The pathogenesis and pathology of ocular onchocerciasis. IV. The pathology. Am $\mathcal{f}$ Ophthalmol 1960; 49: 560-94.

38 Fuglsang H, Anderson J, Marshall TF de C. The head nodule and ocular onchocerciasis in Africa. Tropenmed Parasitol 1976; 27: 355-64.

39 Fuglsang $\mathrm{H}$, Anderson J. Further observations on the relationship between ocular onchocerciasis and the head nodule, and on the possible benefit of nodulectomy. $B r F$ ule, and on the possible be

40 Dadzie KY, Remme J, De Sole G. Epidemiological impact of vector control II. Changes in ocular onchocerciasis. Acta Leidensa 1990; 59: 127-39.

41 Vingtain P, Thillaye B, Karpouzas I, Faure JP. Longitudinal study of microfilarial infestation and humoral immune response to filarial and retinal antigens in onchocerciasis patients treated with ivermectin. Abstract. Ophthalmic Res

42 Cooper PJ, Guderian RH, Proaño R, Taylor DW. Absence of cellular responses to a putative autoantigen in onchocercal chorioretinopathy. Invest Ophthalmol Vis Sci (in press). 